\title{
Value of the bipolar lead CM5 in electrocardiography
}

\author{
A A QUYYUMI, T CRAKE, L J MOCKUS, C A WRIGHT, A F RICKARDS, \\ K M FOX
}

From the National Heart Hospital, London

SUMMARY Only bipolar lead recordings are available during ambulatory monitoring. Their sensitivity in detecting ST segment changes in relation to standard electrocardiographic leads is not known. The magnitude and direction of ST segment changes in the bipolar lead CM5 were compared with those in standard electrocardiographic leads in patients during exercise testing and percutaneous transluminal coronary angioplasty. Thirty patients with coronary artery disease were studied during exercise tests in which ST segment depression $(>0.5 \mathrm{~mm})$ occurred in one or more standard electrocardiographic leads and 13 patients were studied during angioplasty that resulted in ST segment change in one or more leads (I, II, III, V2, V5, and CM5). Lead CM5 was the most sensitive lead $(93 \%)$ during exercise testing and also showed the greatest magnitude of ST segment change below the isoelectric line in $93 \%$ of the patients. Only two patients, one with ST segment elevation in inferior leads and one with changes restricted to septal leads, had no ST segment depression in lead CM5. When ST segment shift from the baseline electrocardiogram was measured the magnitude of depression was greatest in lead CM5 in only $63 \%$ of the patients. During angioplasty of the left anterior descending coronary artery, lead CM5 showed ST segment depression in seven patients, ST segment elevation in two, and a biphasic response in one. Two of the three patients with balloon inflation in the right coronary artery developed ST segment elevation in lead CM5.

Thus lead CM5 is a reliable lead for detecting subendocardial ischaemia experienced during everyday activities in anginal patients. During total occlusion of coronary arteries (as in variant angina or myocardial infarction) lead CM5 commonly shows ST segment depression and changes due to right coronary artery occlusion may not be detected.

Ambulatory monitoring is increasingly being used to detect ST segment changes. $^{1-3}$ Most modern systems provide two channel recordings only, which are obtained from bipolar leads and one of the leads most commonly recorded is CM5. It is, therefore, important to determine the value of lead CM5 compared with that of other conventional electrocardiographic leads used in the detection of ST segment changes, especially to see whether there are specific patterns of ST segment change during conventional 12 lead electrocardiography which would remain undetected if CM5 alone were used. Using exercise testing as a model of subendocardial ischaemia and transluminal angioplasty as a model

Requests for reprints to Dr K M Fox, National Heart Hospital, Westmoreland Street, London W1M 8BA.

Accepted for publication 27 May 1986 of transmural ischaemia, we studied the ST segment changes in lead CM5 and compared them with those in conventional electrocardiographic leads.

\section{Patients and methods}

\section{PATIENTS}

Two groups of patients were studied. Group 1 consisted of 30 patients, 24 men and six women (mean (SD) age 54 (9) years) who were undergoing routine angiocardiography for chest pain. All patients had important coronary artery disease, defined as $>70 \%$ luminal narrowing of one or more major coronary arteries and ST segment depression $>0.5 \mathrm{~mm}$ at the end of exercise. Nine patients had single vessel coronary artery disease, nine had two vessel disease, and 12 had three vessel disease. Patients were exercised after giving informed consent. Seven patients were on $\beta$ adrenoceptor antagonist treatment. Group 2 
consisted of 13 patients who underwent coronary angioplasty; 10 had proximal left anterior descending coronary artery stenosis only and three had single right coronary artery stenosis. There were 10 men and three women (mean (SD) age 52 (8) years). Continuous six lead electrocardiographic recording was performed during percutaneous transluminal coronary angioplasty and all patients had ST segment changes in at least one electrocardiographic lead.

\section{EXERCISE TESTING}

Group 1 patients performed a maximal symptom limited exercise test. A 12 lead electrocardiogram was recorded at the end of each stage and lead CM5 was substituted for lead aVR. The patients exercised on a bicycle ergometer at 50 revolutions per minute and the workload was increased every three minutes so that there was an increase in heart rate of at least 10 beats a minute at each stage. The tests were stopped when anginal chest pains, frequent ventricular extrasystoles, important ST segment depression $(>3 \mathrm{~mm})$, a fall in blood pressure, or exhaustion developed.

The electrocardiogram was recorded on an ink jet recorder (Siemens Mingocard 7). The indifferent electrode of lead CM5 was placed over the manubrium and the active electrode was on the precordial V5 position. ST segment change was measured accurately by means of a magnifying lens with a graticule marked in $0.1 \mathrm{~mm}$ divisions (Flubacher, Switzerland). A line drawn between two consecutive PR segments gave the zero baseline. ST segment change was measured $0.08 \mathrm{~s}$ after the $\mathrm{J}$ point.

\section{ELECTROCARDIOGRAPHIC RECORDING DURING ANGIOPLASTY}

In group 2 patients a continuous six lead electrocardiogram (I, II, III, CM5, V2, and V5) was recorded with the patient in the supine position before, during, and after balloon inflation. ST segment change was measured with a magnifying lens as described above and only patients with ST segment depression or elevation of $\geqslant 0.5 \mathrm{~mm}$ from the baseline electrocardiogram were included in this study.

\section{Results}

COMPARISON OF CONVENTIONAL

ELECTROCARDIOGRAPHIC LEADS WITH LEAD CM5 DURING EXERCISE TESTING

Twenty eight $(93 \%)$ of 30 group 1 patients developed $>0.5 \mathrm{~mm}$ ST segment depression below the isoelectric line in lead CM5 and this was the most sensitive of the 12 leads monitored in this study. In comparison, ST segment depression of $>0.5 \mathrm{~mm}$ occurred in $70 \%$ of patients in lead V6, $67 \%$ in lead V5, $43 \%$ in lead V4, and $50 \%$ in lead II (table). In $93 \%$ of patients lead CM5 also showed the greatest depression of the ST segment below the isoelectric line (table). In the remaining two patients, lead V5 and $\mathrm{V} 2$ respectively demonstrated the maximum ST segment depression. In these patients, although ST segment depression occurred in lead CM5, it was $<0.5 \mathrm{~mm}$. ST segment depression was $1.4(0.9)$ (mean (SD)) in lead CM5 in the 28 patients in whom important depression developed. In comparison the magnitude was $1.2(0.5) \mathrm{mm}$ (mean (SD)) in the 21 patients with important depression in lead V6 and $1.45(0.6)$ in 20 patients with important depression in lead V5. Only 25 patients fulfilled the conventional criterion of $1 \mathrm{~mm}$ ST segment depression $0.08 \mathrm{~s}$ after the J point at the end of exercise in one or more leads. Lead CM5 showed the maximum ST segment depression in $23(92 \%)$ of these 25 patients.

In 17 patients ST segment depression was observed in two or more of the inferior leads (II, III, $\mathrm{aVF}$ ) as well as the anterolateral leads (V4, V5, V6). Of the remaining 13 patients, 11 had changes in the

Table Number of patients who developed ST segment depression of varying magnitude from the isoelectric line in the 12 electrocardiographic leads during bicycle ergometry

\begin{tabular}{|c|c|c|c|c|c|}
\hline \multirow[b]{2}{*}{ Leads } & \multicolumn{4}{|c|}{ Patients with $S T$ depression } & \multirow{2}{*}{$\begin{array}{l}\text { Maximum } \\
\text { depression }\end{array}$} \\
\hline & $\geqslant 2 \mathrm{~mm}$ & $<2-\leqslant 2 \mathrm{~mm}$ & $>0.5-1.0 \mathrm{~mm}$ & $<0.5 \mathrm{~mm}$ & \\
\hline $\begin{array}{l}\text { I } \\
\text { II } \\
\text { III } \\
\text { aVL } \\
\text { aVF } \\
\text { V1 } \\
\text { V2 } \\
\text { V3 } \\
\text { V4 } \\
\text { V5 } \\
\text { V6 } \\
\text { CM5 }\end{array}$ & $\begin{array}{l}0 \\
1 \\
0 \\
0 \\
0 \\
0 \\
1 \\
1 \\
1 \\
2 \\
1 \\
5\end{array}$ & $\begin{array}{r}1 \\
8 \\
3 \\
0 \\
4 \\
0 \\
1 \\
1 \\
6 \\
10 \\
7 \\
18\end{array}$ & $\begin{array}{r}4 \\
6 \\
4 \\
0 \\
6 \\
0 \\
1 \\
3 \\
6 \\
8 \\
13 \\
5\end{array}$ & $\begin{array}{l}1 \\
8 \\
5 \\
5 \\
5 \\
0 \\
0 \\
0 \\
2 \\
2 \\
3 \\
2\end{array}$ & $\begin{array}{r}0 \\
0 \\
0 \\
0 \\
0 \\
0 \\
1 \\
0 \\
0 \\
1 \\
0 \\
28\end{array}$ \\
\hline
\end{tabular}



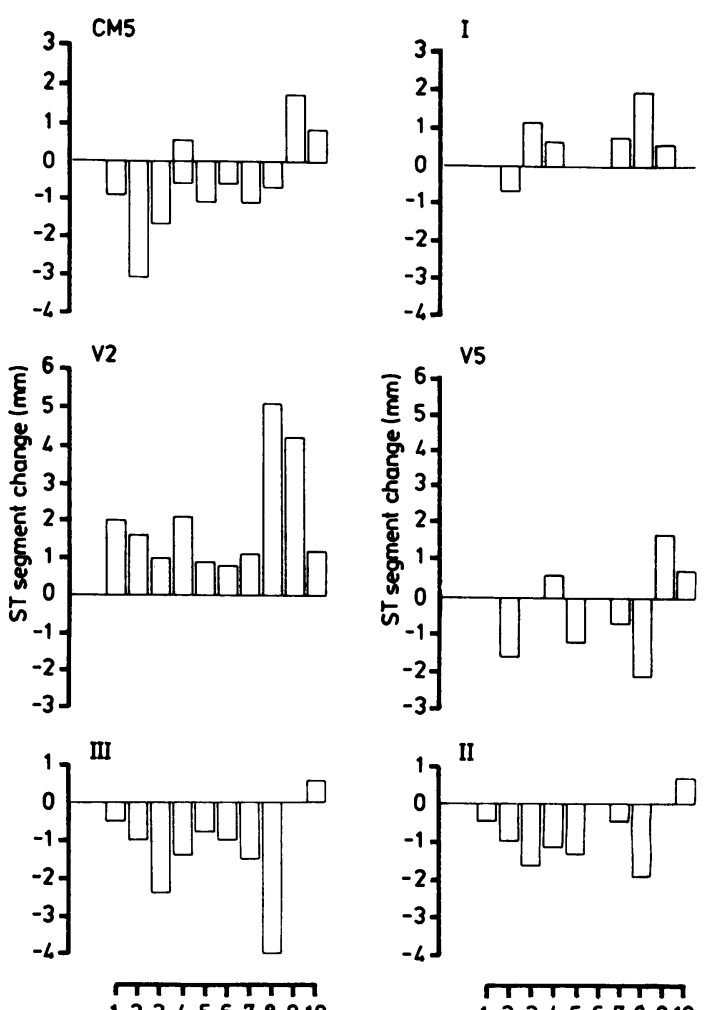

12365678910

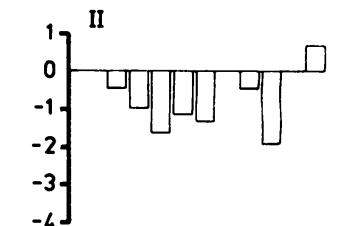

T21 T1717

Patients

Fig 1 Magnitude and direction of ST segment changes in lead CM5 and other standard electrocardiographic leads in 10 patients with single vessel disease during balloon inflation in the left anterior descending coronary artery.

anterolateral leads only, one in leads V2 and V3 only, and another in lead II only. Only one patient with an old inferior myocardial infarction developed ST segment elevation in inferior leads during exercise. All other patients either showed depression or had no shift of the ST segment with exercise. The patient with ST segment elevation in the inferior leads during exercise showed a smaller change in lead CM5 than in leads V5 and V6.

When ST segment shift from the baseline electrocardiogram was examined instead of the shift from the isoelectric line as described above, lead CM5 showed greater or equal magnitude of ST segment depression compared with standard electrocardiographic leads in $19(63 \%)$ patients. In none of the patients was ST segment change confined to the inferior leads. Even the two patients with isolated right coronary artery stenoses had electrocardiographic changes in leads V5 and V6 in addition to changes in the inferior leads.
COMPARISON OF CONVENTIONAL

ELECTROCARDIOGRAPHIC LEADS WITH CM5

DURING ANGIOPLASTY

During left anterior descending coronary artery occlusion leads V2 and CM5 were the most sensitive in detecting ST segment change (fig 1). In lead CM5 seven patients had ST segment depression, two had ST segment elevation, and one patient had ST segment depression progressing to ST segment elevation during balloon inflation (fig 1). Changes in the ST segment occurred approximately at the same time in all the leads in individual patients and the mean (SD) duration of onset was 15 (12)s after balloon inflation.
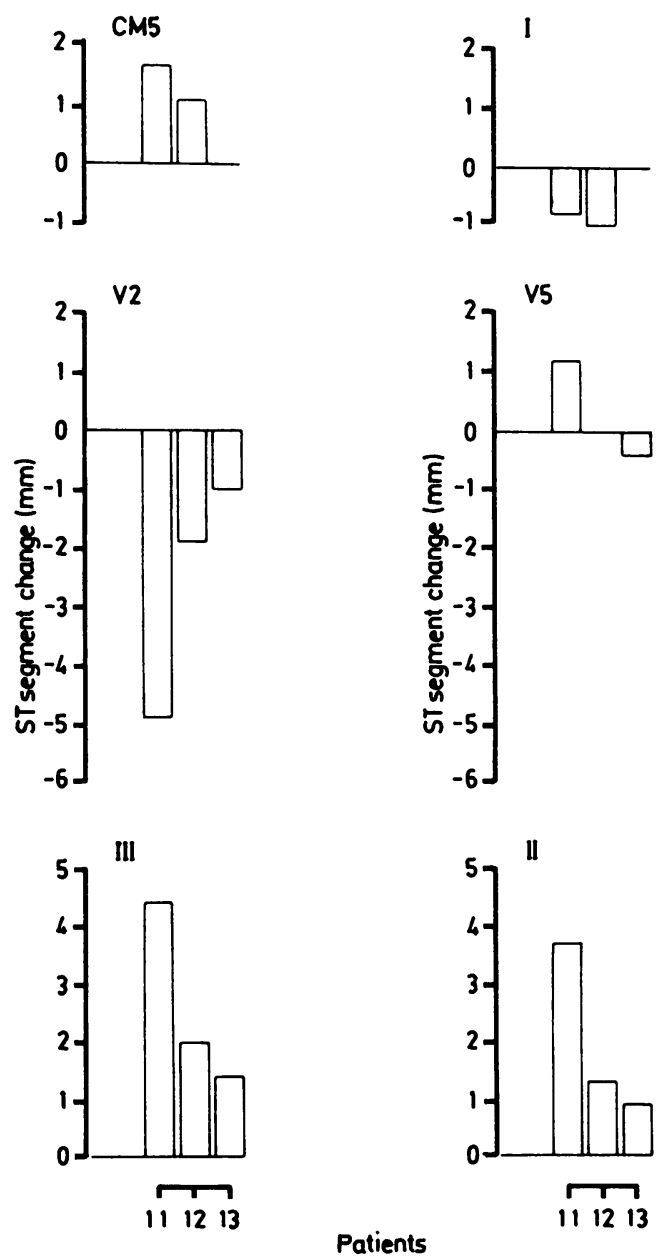

Fig 2 Magnitude and direction of ST segment changes in the lead CM5 and other standard electrocardiographic leads in three patients with single vessel disease during balloon inflation in the right coronary artery. 
During balloon occlusion of the right coronary lumen in three patients, ST segment elevation occurred in the inferior leads II and III and this was accompanied by ST segment depression in lead CM5 in two patients (fig 2).

\section{Discussion}

Multiple lead electrocardiography is more sensitive than single lead systems for detecting coronary artery disease by stress testing. ${ }^{4-6}$ The addition of lead CM5 increases the sensitivity further. ${ }^{46}$ When only bipolar lead analysis is available, however, as in ambulatory ST segment monitoring it is important to determine which lead or leads are most likely to demonstrate ST segment change. Although in this study we have confirmed that lead CM5 is the most sensitive electrocardiographic lead during exercise testing, ${ }^{46}$ further information is required on its usefulness in relation to conventional electrocardiographic leads in various clinical situations. For this purpose we selected patients who had ST segment depression during stress testing in at least one of the standard electrocardiographic leads and correlated the changes observed in lead CM5 with changes in the other leads. We thus attempted to identify patients in whom important ST segment changes occur during multiple lead electrocardiography but are not reflected in lead CM5.

There are two common patterns of myocardial ischaemia. Angina on exertion usually results from subendocardial ischaemia and causes ST segment depression on the surface electrocardiogram, a situation reproduced during exercise testing. Transmural ischaemia may result from severe coronary spasm or thrombotic occlusion of a major coronary vessel, and balloon inflation in the coronary artery will have a similar effect. Ischaemia results in ST segment elevation in the territory of the artery occluded. We evaluated the usefulness of ST segment changes in lead CM5 in these two situations. We found that ST segment changes in lead CM5 occurred in $93 \%$ of all patients who were exercised and in $100 \%$ of patients who developed anterolateral and inferior wall ischaemia during exercise. It was only unreliable in a patient with previous inferior wall infarction and ST segment elevation during exercise and in another patient who developed changes in leads V2 and V3 only. During angioplasty, complete occlusion of the coronary lumen of the left anterior descending coronary artery resulted in ST segment change in all patients in lead CM5. The direction of change was variable, however, and occasionally bidirectional. With right coronary artery occlusion, one of the three patients did not develop significant ST segment change in lead CM5 although there was ST segment elevation in the inferior leads (fig 2).

Depression below the arbitrary isoelectric line is conventionally used in the visual analysis of ST segments. By this criterion the greatest ST segment depression was found in lead CM5 in $93 \%$ of the patients. When the shift of the ST segment from the resting electrocardiogram was analysed, in $39 \%$ of the patients the change was greater in leads other than CM5. Thus, although the depression of ST segments may be more obvious in lead CM5, the rate of ST segment depression is often greater in other electrocardiographic leads which do not demonstrate the greatest magnitude of ST segment depression below the isoelectric line.

There were no significant differences between the magnitude of ST segment depression in lead CM5 and the presence or absence of inferior ST segment depression. In one patient, however, in whom there was ST segment elevation in the inferior leads on exercise and $>1 \mathrm{~mm}$ ST segment depression in lead V5, lead CM5 showed unimportant $(<0.5 \mathrm{~mm}) \mathrm{ST}$ segment depression. It has been suggested that lead CM5 has components from both inferior and anterolateral myocardial walls ${ }^{7}$ and if differing directional changes occur in these two areas, there may be little change in lead CM5. This is borne out to some extent by the electrocardiographic changes observed during angioplasty of the left anterior descending coronary artery. Here lead III often showed ST segment depression and lead V2 had ST elevation and lead CM5 demonstrated ST depression, ST elevation, or a biphasic response.

Thus although CM5 is a reliable lead for detecting the common type of exertional ischaemia in most patients, it may not detect ischaemia in those with previous inferior myocardial infarction and where changes are restricted to anteroseptal (V1, V2, V3) leads. In patients with coronary spasm, in the left anterior descending or right coronary arteries the change in lead CM5 is usually ST segment depression but is sometimes bidirectional; therefore the absence of ST segment elevation in lead CM5 does not imply absence of occlusion of the coronary arteries. Furthermore, in this situation, lead CM5 often does not demonstrate the maximum ST segment shift and may not be the ideal lead (figs 1 and 2). When spasm is present a bipolar lead CM2 may be more suitable for detecting septal ST segment changes and a bipolar lead resembling lead II $^{46}$ may be more suitable for detecting inferior wall ischaemia.

Therefore, if two bipolar leads are available for ambulatory monitoring, CM5 would certainly be the most useful first lead. The choice of the second lead should depend on other clinical factors such as 
376

detection of spasm or demand angina and electrocardiographic factors such as the presence of an old inferior infarction. Use of bipolar leads resembling those in which the exercise test is most positive would also improve the sensitivity of the technique.

\section{References}

1 Quyyumi AA, Mockus L, Wright C, Fox KM. Morphology of ambulatory ST segment changes in patients with varying severity of coronary artery disease: investigation of the frequency of nocturnal ischaemia and coronary spasm. $B r$ Heart $\mathcal{f}$ 1985;53:186-93.

2 Deanfield J, Wright C, Fox K. Treatment of angina pectoris with nifedipine: the importance of dose titration. Br Med F 1983;286:1467-70.
Quyyumi, Crake, Mockus, Wright, Rickards, Fox

3 Schang SJ, Pepine CJ. Transient asymptomatic ST segment depression during daily activity. $A m \mathcal{f}$ Cardiol 1977;39:396-400.

4 Chaitman BR, Bourassa MG, Wagniart P, Corbara F, Ferguson RJ. Improved efficiency of treadmill exercise testing using a multiple lead ECG system and basic hemodynamic exercise response. Circulation 1978;57:71-9.

5 Mason RE, Likar I, Biern RO, Ross RS. Multiple-lead exercise electrocardiography. Experience in 107 normal subjects and 67 patients with angina pectoris, and comparison with coronary cinearteriography in 84 patients. Circulation 1967;36:517-25.

6 Chaitman BR, Waters DD, Bourass MG, Tuban JF, Wagniart P, Ferguson RJ. The importance of clinical subsets in interpreting maximal treadmill exercise test results: the role of multiple-lead ECG systems. Circulation 1979;59:560-70.

7 Froelicher VF Jr, Wolthius R, Keiser N, et al. A comparison of two bipolar exercise electrocardiographic leads to lead V5. Chest 1976;70:611-6. 